\title{
Towards a public, standardized, diagnostic benchmarking system for land surface models
}

\author{
G. Abramowitz \\ University of New South Wales, Sydney, Australia \\ Correspondence to: G. Abramowitz (gabriel@unsw.edu.au) \\ Received: 2 February 2012 - Published in Geosci. Model Dev. Discuss.: 20 February 2012 \\ Revised: 14 May 2012 - Accepted: 15 May 2012 - Published: 5 June 2012
}

\begin{abstract}
This work examines different conceptions of land surface model benchmarking and the importance of internationally standardized evaluation experiments that specify data sets, variables, metrics and model resolutions. It additionally demonstrates how essential the definition of a priori expectations of model performance can be, based on the complexity of a model and the amount of information being provided to it, and gives an example of how these expectations might be quantified. Finally, the Protocol for the Analysis of Land Surface models (PALS) is introduced - a free, online land surface model benchmarking application that is structured to meet both of these goals.
\end{abstract}

\section{Introduction}

Land surface models (LSMs) simulate the exchange of water, heat and carbon between the land surface and atmosphere, and represent these processes within climate models. Climate models in turn have evolved from extremely simplified tools used to gain a conceptual understanding of broad-scale climate features - such as continental boundary effects (e.g. Manabe, 1969) - to something more akin to operational weather forecasting tools. Climate projections now inform multi-million dollar decisions, and this is reflected in the pressures that research scientists face to provide a "comprehensive representation of the four major components of the climate system" (Gordon et al., 2002) "for simulating past, present, and future climates" (Collins et al., 2006). This change of focus has driven a commensurate transition in the nature of model evaluation from qualitative to quantitative analysis.
While LSM evaluation increasingly relies on a broad collection of data sources (e.g. in-situ gas exchange measurements, streamflow and satellite-based measurements), the limited nature of their availability and quality control historically has meant that the transition from qualitative analysis in LSM evaluation has been ad hoc. Although the term "benchmarking" has recently increased in popularity in the LSM community (e.g. Abramowitz et al., 2008; Blyth et al., 2011), there is apparent confusion regarding its meaning. In its weakest and perhaps most common usage, benchmarking is simply synonymous with model evaluation of any sort, and so apparently only reflects a change in language rather than practice. Benchmarking has also been used to refer to a single institution's LSM evaluation program (e.g. Blyth et al., 2011), which would usually define previous model versions as the performance standard. The third usage, and one that is discussed in Sect. 2, defines benchmarking as a coordinated effort to define community-wide reference data sets, spatial and temporal resolutions, variables and metrics for evaluation. Here these are referred to as standardized experiments.

Section 3 illustrates the importance of an additional constraint on standardized experiments - the a priori specification of expectations of model performance. That is, given the complexity of a model, and the amount of information provided to it in its time-independent parameters and timedependent input variables, how well should we expect it to perform? One possible answer to this question that recognizes that some environments are more difficult to simulate than others is also discussed in Sect. 3. This solution is then used to show how one might construct a hierarchy of performance benchmarks that could be used to rank models.

Finally, in Sect. 4, the Protocol for the Analysis of Land Surface models (PALS) is introduced, a web-based LSM 
Table 1. A collection of commonly ignored model development issues that affect the reliability, useability and reproducibility of LSMs, represented by two caricaturized models.

\begin{tabular}{ll}
\hline The good & The ugly \\
\hline Model has technical documentation & Model has no technical documentation \\
$\begin{array}{l}\text { Technical documentation matches what is in the model } \\
\text { code }\end{array}$ & $\begin{array}{l}\text { Technical documentation related to what was in the } \\
\text { code of previous model versions }\end{array}$ \\
$\begin{array}{l}\text { Model is open source, community oriented and has hun- } \\
\text { dreds of users }\end{array}$ & $\begin{array}{l}\text { Model is proprietary and only used by a few people in } \\
\text { one organisation }\end{array}$ \\
$\begin{array}{l}\text { All development of the model is contained in a version } \\
\text { control system }\end{array}$ & $\begin{array}{l}\text { Individuals maintain and manage multiple versions in } \\
\text { home directories/desktop }\end{array}$ \\
$\begin{array}{l}\text { Model has a clear user interface and user guide } \\
\text { Code is clearly commented, and logically structured }\end{array}$ & $\begin{array}{l}\text { Model has no user guide and no specific interface } \\
\text { Code is not commented at all and structure is ad hoc } \\
\text { Variable names are consistent throughout the code and } \\
\text { relate to their function }\end{array}$ \\
$\begin{array}{l}\text { Model changes meet prescribed } \\
\text { performance/realism/functionality checks }\end{array}$ & $\begin{array}{l}\text { meaningless } \\
\text { Changes are accepted purely on the basis of personal } \\
\text { preference }\end{array}$ \\
\hline
\end{tabular}

evaluation tool that is structured to meet these goals. It acts both as a data set repository and automated evaluation tool, to be used as either a model development facility or framework for model comparison experiments, and keeps a complete version history of all the data it contains.

While the discussion is focused on LSMs designed for use in high resolution climate model simulations, note that much of what is presented here is equally applicable to hydrological modelling or ecological modelling in areas where appropriate data sources are available.

\section{Benchmarking using internationally standardized experiments}

The benefits of internationally accepted standard experiments - prescribing LSM driving data, evaluation data, variables, metrics and possibly surface parameter information - are many. They allow different research teams to immediately compare results, identify shared weaknesses or strengths in LSMs and provide a fast cost-benefit analysis of any proposed modifications to a modelling system. Equally important, this definition of benchmarking minimizes the potentially very serious impact of the seemingly trivial modelling problems shown in Table 1 (where the two columns represent polarized representations of model development). These issues, while well recognized in commercial software development, are relatively new to researchers working in science, where funding sources and performance metrics rarely, if ever, recognize the importance and resource requirements associated with model development and management. One might speculate that the increasingly operational nature of climate projection will mean that these standards, so essential in other software development environments, cannot continue to be ignored by research managers in the future.

To gauge the importance of the model traits in Table 1, try asking yourself which of these two caricaturized models is: more likely to be reliable; more likely to contain critical bugs; more likely to be used inappropriately; and more like the model you use? It seems clear that a benchmarking environment defined and maintained by a single research group is more likely to allow coding bugs or unrecognized weaknesses to propagate through successive model generations than an internationally agreed benchmarking system where evaluation against other LSMs is commonplace.

By sharing the investment required in benchmarking experiments, an internationally defined benchmarking experiment set also allows a greater depth of LSM analysis as shared experiments accrue. The process of defining this type of benchmark for the LSM community is the goal of the International Land Model Benchmarking (ILAMB) group (www.ilamb.org).

\section{Benchmarking using a priori expectations of performance}

An equally important aspect of LSM benchmarking, and one that is rarely addressed, is an assessment of the level of performance we should expect of LSMs. Given a variable, spatial scale, temporal scale and metric, can we specify a priori how close a model should be to observations? Put simply, what constitutes a "good" model?

For a single variable and metric, intuition might suggest that choosing the "best" model is easy - it performs best in 
the given metric. Yet there are several critical caveats to this response that indicate it is not a satisfactory answer to what defines a "good" model. If nothing else, it rules out the very real possibility that the "best" model is in fact a poor model. Ginzburg and Jensen (2004) give the excellent example of Ptolemy's epicycle model explaining the motion of the solar system's planets through the night sky as well as Newtonian mechanics, despite its absurd physical representation. Below are four criteria by which to judge a good model. Performance is just one of these:

1. The simplicity of a model. This criterion is essentially the principle of parsimony or Occam's razor - a simpler model is preferred to a complicated model where they perform to a similar standard. Simpler in this case can refer to the functional representation of relationships between quantities or the number of internal parameters. Simpler, more succinct models are preferred, as they are easier to understand and diagnose when they behave in unexpected ways.

2. The amount of information provided to a model. A model that requires fewer time-dependent driving variables and fewer parameters describing its operating environment is preferred over one that requires more, where it performs to a similar standard. It should be clear that (1) and (2) are both essentially principles of parsimony, applied to different aspects of modelling. The motivation for their separation will be made clear below.

3. Identifiability or physical representativeness of a model. A model that is physically-based is preferred to one that is statistically-based. The internal variables of a purely empirical model (such as a feed-forward neural network) need not bear any resemblance to variables measurable in the physical system. At the other end of the spectrum, a truly physically-based model's variables are so closely aligned with those in the system that it requires no calibration whatsoever. In most practical circumstances however, the distinction between these two is quite subtle. An apparently physically-based model whose internal variables purport to be quantities associated with the physical system must be considered at least partly empirical in circumstances when these variables are unmeasured or unmeasurable. In this case a calibration data set is used to tune the model to the time, location and circumstances of the calibration data set, rendering it at least partly empirically-based.

4. How well a model performs out of sample. Model performance in given metric must be assessed out of sample. That is, the data used to assess the model must not have been used in the model's calibration or development. Performance on calibration data should not be used for evaluation.
A "good" model therefore need not be the best performing model - it may be "good" because of it's ability to provide adequate simulations with very little input data, the simplicity of its algorithms, or the ability of its constituent variables to be unambiguously identified with those in the natural system it simulates. A priori expectations of performance in outof-sample experiments should in some way take these considerations into account. One should have lower expectations of a simple model than of a complicated one. One approach to doing this that explicitly considers three of these four criteria is empirical benchmarking (e.g. Abramowitz et al., 2008). This essentially involves training an entirely empirical model (such as a regression or neural network based approach) to do the job of a LSM, and testing the empirical model out of sample on the LSM evaluation data set. One can then manipulate which input variables the empirical model uses, as well as its complexity, in order to gauge the level at which a LSM is performing.

An example is shown in Fig. 1. It shows a smoothed fouryear time series of latent heat flux (LH) at a single flux tower site (Tumbarumba; see Leuning et al., 2005). Observations are shown in black and a LSM simulation (the Community Atmosphere Biosphere Land Exchange model (CABLE); see Wang et al., 2011), driven with site meteorology, vegetation type, soil type, reference height and vegetation height, is shown in blue. In most circumstances, an author would suggest this is a competent, even very good, LSM simulation. The two curves are clearly highly correlated and regularly overlap.

The red curve in Fig. 1 shows a simple empirical model simulation of LH. First, a linear regression between downward shortwave radiation (SW) and LH was performed using data from 30 sites globally that did not include the Tumbarumba site - around 2500000 time steps of data. Then, these two regression parameters were used to predict $\mathrm{LH}$ at Tumbarumba, based solely on SW, at a half-hourly time step. From Fig. 1 it can be seen that this extremely simple empirical model benchmark has low variance, as we might expect from a linear regression model (see the minimum value, maximum value and standard deviation of the observed, modelled and benchmark time series, respectively, in upper centre of Fig. 1). It nevertheless outperforms the LSM in root mean square error (not shown) and normalized mean error (NME, shown in Fig. 1), both in the smoothed time series shown in Fig. 1 ("Score_smooth") and the original half-hourly time series ("Score_all").

An identically structured empirical model is used in Fig. 2 to predict net ecosystem exchange of $\mathrm{CO}_{2}$ (NEE), again only as a function of SW. It shows the average diurnal cycle of NEE across several years of a single site, divided into four seasonal panels. We again see that a commonly used qualitative metric, this time average diurnal cycle, appears convincingly simulated by a simple regression model, with the NME values reflecting this in all seasons. In these two metrics, at 


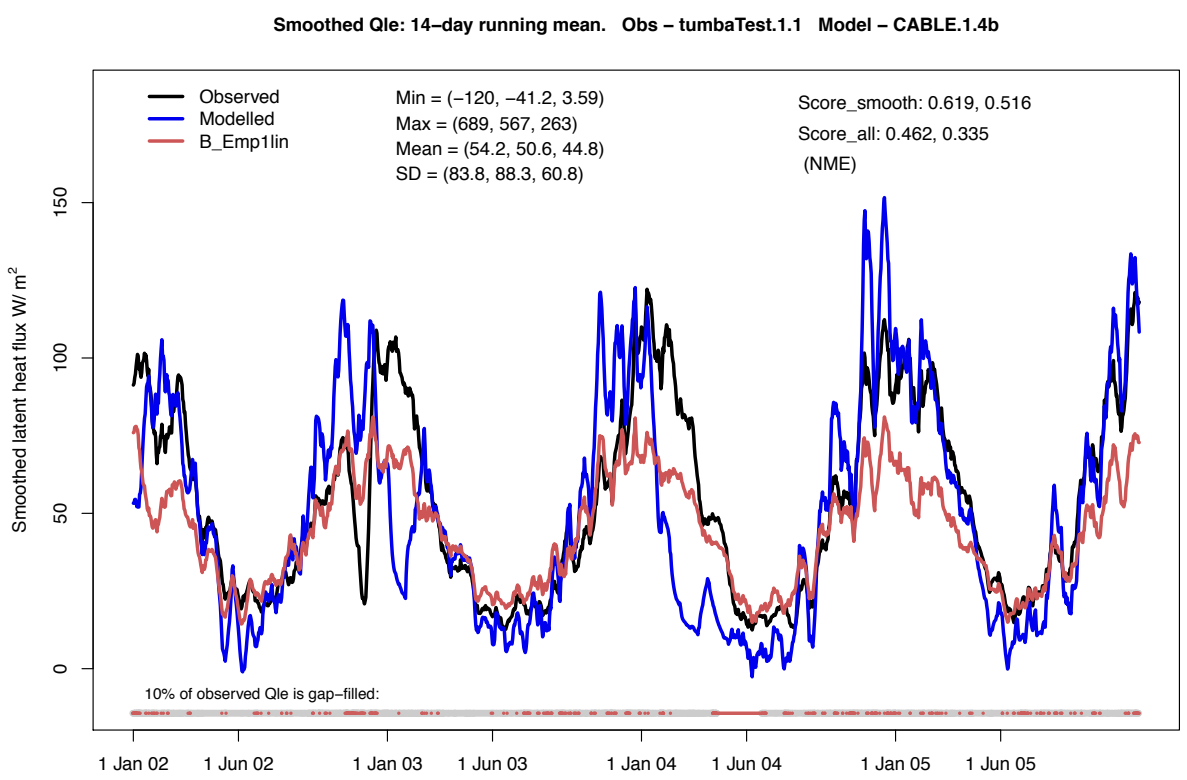

Fig. 1. A 14-day running mean latent heat flux time series at a single flux tower site. While model performance (blue) relative to observations (black) look very good, many metrics on this time series show that an out-of-sample linear regression (red) of latent heat against short-wave radiation performs similarly.

least in this instance, this LSM is performing comparably to a linear regression against sunlight.

Not all examples are this revealing of course; these were deliberately chosen to highlight the utility of this approach, but they do also illustrate the importance of what we might call a priori benchmarking. Qualitative similarity between modelled and observed curves, so often passed as rigorous model evaluation, may in fact tell us very little about model performance. Using an empirical model in this way reveals: the extent to which LH is predictable from SW alone; how a very simple functional relationship appears in familiar diagnostic measures; and how predictable LH is, out-of-sample, at the Tumbarumba site. Since empirical model performance will be poorer at sites that exhibit unusual or unique behaviour, this approach implicitly recognizes that some environments are more difficult to simulate than others. It also gives a model-like time series and so can provide a benchmark level of performance in any chosen metric.

Using this approach, a hierarchy of benchmarks can be constructed and used to assess how well a model is performing relative to its complexity and the amount of information provided to it in its inputs and parameters. By making a comparison similar to that shown in Figs. 1 and 2, using empirical models that vary in their complexity and the variables that are provided to them, we can rank a LSM's performance. Figure 3 gives an example. It shows probability density functions of sensible heat flux (SH) as observed (black) at Tumbarumba, as simulated by a LSM (blue), and as predicted by three increasingly complex empirical models (red, yellow, green). These empirical models are: (1) the linear regression against SW discussed above; (2) a multiple linear regression of SH against both SW and surface air temperature $(T)$; and (3) a k-means clustering of the time series of SW, $T$ and wind speed $(W)$, with a multiple linear regression between $(\mathrm{SW}, T, W)$ and $\mathrm{SH}$ performed at each cluster. In this example, 243 clusters were used. This simply creates a piecewise linear functional representation of the relationship between ( $\mathrm{SW}, T, W)$ and $\mathrm{SH}$ in the training data set. More generally, this hierarchy of benchmarks could also use energy conservation and observational uncertainty as part of its definition, as illustrated in Table 2.

Flux tower data was chosen here for three reasons. Firstly, it allows the construction of an empirical model that operates at the same time step size and using the same input data as the LSM. The types of functional relationships between inputs and outputs seen in the empirical model should therefore be very similar to those of the LSM. Next, flux tower data has directly measured meteorological drivers at the same time and spatial scale as the measured fluxes used for evaluation. While there are significant uncertainties associated with flux tower data, particularly surrounding energy conservation (e.g. Wilson et al., 2002; Kidston et al., 2010), using coincident driving and evaluation products that involve little or no interpolation or additional modelling means that this data source offers unparalleled model constraint. We are as close as is possible with current data availability to having error free driving data and so as close as is currently possible to true diagnostic model evaluation. Finally, flux towers are one of the very few data sources that provide data in quantities that allow for the construction of robust empirical models. 

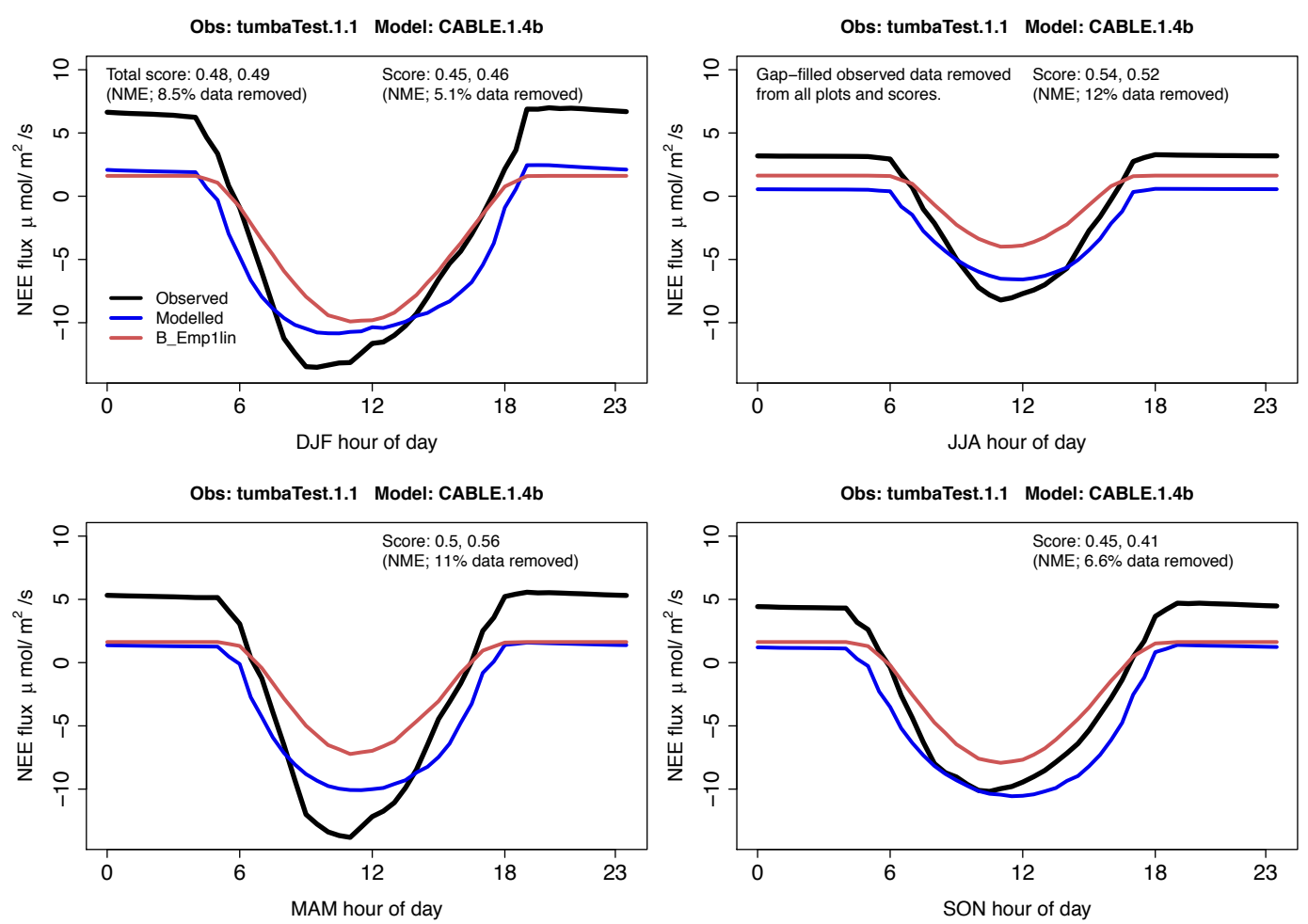

Fig. 2. Average diurnal cycle of net ecosystem exchange of $\mathrm{CO}_{2}$ (NEE) at a single flux tower site, shown in a separate panel for each season. As in Fig. 1, an out-of-sample linear regression of NEE against downward shortwave radiation (shown in red) performs comparably to a LSM in this instance (blue). Normalized mean error of the average diurnal cycle is used as the scalar metric, shown separately for each panel and combined in the DJF panel.

Table 2. A hierarchy of a priori levels of benchmark performance for LSMs, with tiers defined by increasingly complex empirical models provided with more meteorological and site description variables.

Conservation of mass and energy (weakest)
Linear regression against shortwave radiation (weak)
$\ldots \ldots \ldots \ldots \ldots$
Complex empirical model as a function of meteorology and vegetation and soil type (strong)
Model output within observational uncertainty ranges (strongest)

While the results above use 30 flux tower sites (around 2500000 model time steps), the La Thuile Fluxnet release contains around 500 sites. It is a goal of the PALS project described below to continue to process flux tower data for LSM evaluation as it is made available.

While this approach seems to offer the best option for a priori benchmarking, it is essential to acknowledge that evaluation at flux towers does not by any means constitute complete LSM evaluation. Larger spatial and temporal scale features produced by LSMs in coupled models are a key aspect of climate prediction, and this is undoubtedly the ultimate purpose for most LSMs. These features are, however, emergent properties of LSMs and their atmospheric model counterparts (or forcing data), so untangling cause and effect in circumstances of uncertain or error-prone forcing data can be extremely difficult. Accordingly, model evaluation for the diagnosis of model deficiencies can also be very difficult in this context. While it is also commonly argued that LSMs are designed to simulate grid cells rather than point-scale data, note that LSMs have no explicit length scale, and that LSMs rarely if ever undergo fundamental change when run at different resolutions within a coupled model environment.

The process above gives us an idea of how good a model is relative to its complexity and the information it is provided with, but it cannot answer the somewhat more subjective question how good is good enough? The "validity" of a model relative to a user's needs (e.g. Oreskes et al., 1994; Medlyn et al., 2005) clearly depends on many more factors than the four discussed above. A user may not care that a complex LSM performs on par with a linear regression against meteorological variables if their purpose is simply to resolve diurnal flux cycles. It is perhaps even unusual 


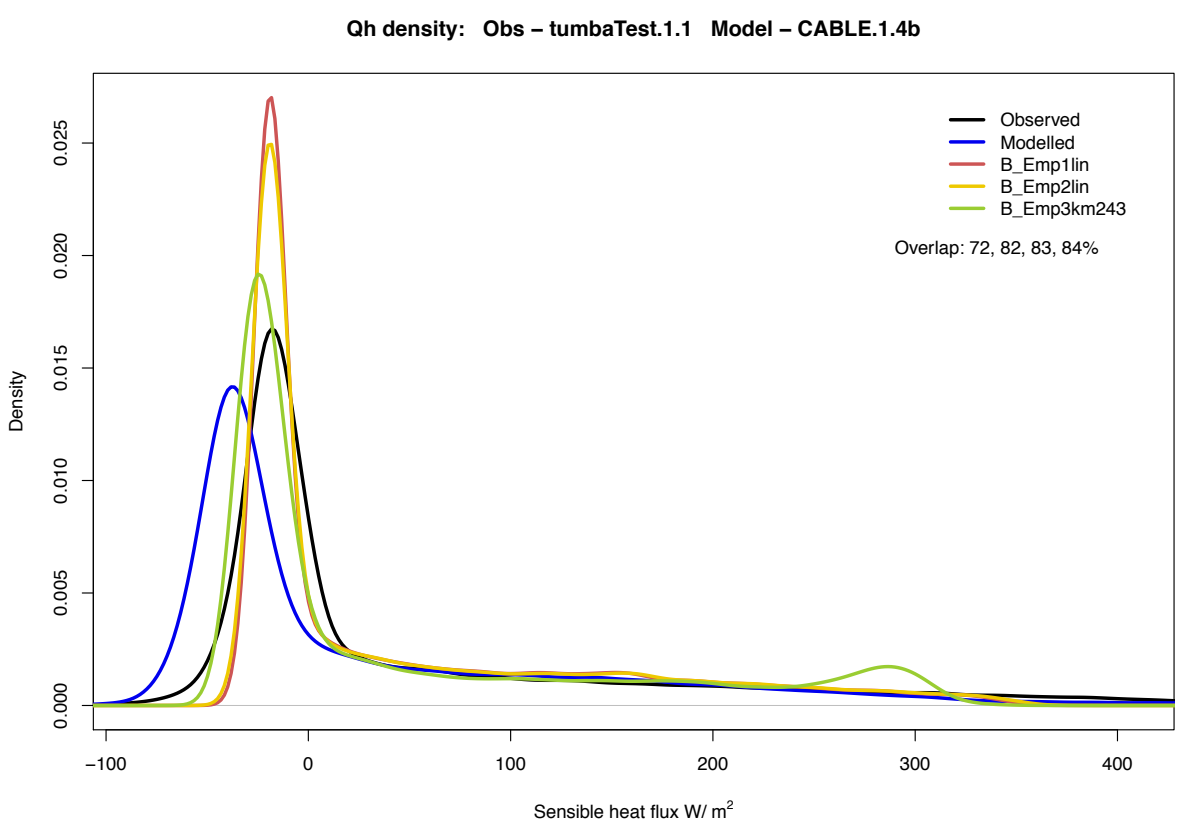

Fig. 3. Probability density functions (PDFs) of observed (black) and modelled (blue) sensible heat flux at a single flux tower site. Three additional PDFs representing a hierarchy of empirical models are also shown. The proportion of overlap of observed and modelled (or a benchmark PDF), expressed as a percentage, is used as the scalar metric for this analysis type. Metric values are listed in the same order as the legend.

that the complexity of a model is tailored to its application. Indeed, this is arguably the state of LSM use within climate models today. While most current generation LSMs have 3040 vegetation and soil description parameters, almost all are provided only with a vegetation type and soil type for each location (typically from a choice of 20 possible types globally). Put differently, the parameter information required for these relatively complex LSMs is not available at the global scale, so parameter values are fitted to effective "types" and calibrated with available data belonging to each type. This over parameterized approach risks calibrating LSMs to the particular variables, metrics, time and spatial scales used in their calibration.

\section{The Protocol for the Analysis of Land Surface models (PALS)}

PALS (pals.unsw.edu.au) is an automated web application for the diagnostic evaluation of LSMs that tries to meet the two goals outlined in the two previous sections. The general structure of the PALS is outlined below before the first phase of implementation and future developments are discussed.

PALS performs several functions simultaneously. First, it acts as a repository for quality controlled, standardizedformat LSM driving and evaluation data sets, and maintains a complete version history of each data set. Subsets of PALS data sets are aggregated into experiment structures, each of which may contain LSM forcing data sets, information for constraining LSM parameters and evaluation data sets.

PALS also allows upload of model output data files associated with a PALS experiment. Each time a LSM output is uploaded, ancillary files associated with it may also be uploaded. For example, one may wish to upload simulation log files, namelist files, control files, parameter files or even the model code associated with a particular simulation as a way of ensuring the reproducibility of a simulation. Unless a user decides to delete their model output contributions to an experiment, PALS will maintain the complete version history of model output experiment submissions.

Once LSM output is uploaded, PALS automatically runs a range of analyses comparing LSM output and observed data. Particular types of analysis are associated with particular types of experiments - examples of analyses associated with a single flux tower based experiment are shown in Figs. 1 through 4 (these images were downloaded directly from PALS). For this to work, of course, LSM output files need to be in a standardized format. PALS currently reads netcdf output files in the Assistance for Land-surface Modelling activities (ALMA; http://www.lmd. jussieu.fr $\sim$ polcher/ALMA/) format (CABLE - Wang et al., 2011; ORCHIDEE - Krinner et al., 2005; JULES - Blyth et al., 2006) as well as CLM's netcdf format (Levis et al., 2004; Oleson et al., 2004). Currently, all automated analyses use R (http://www.r-project.org/) to generate graphics, and the 


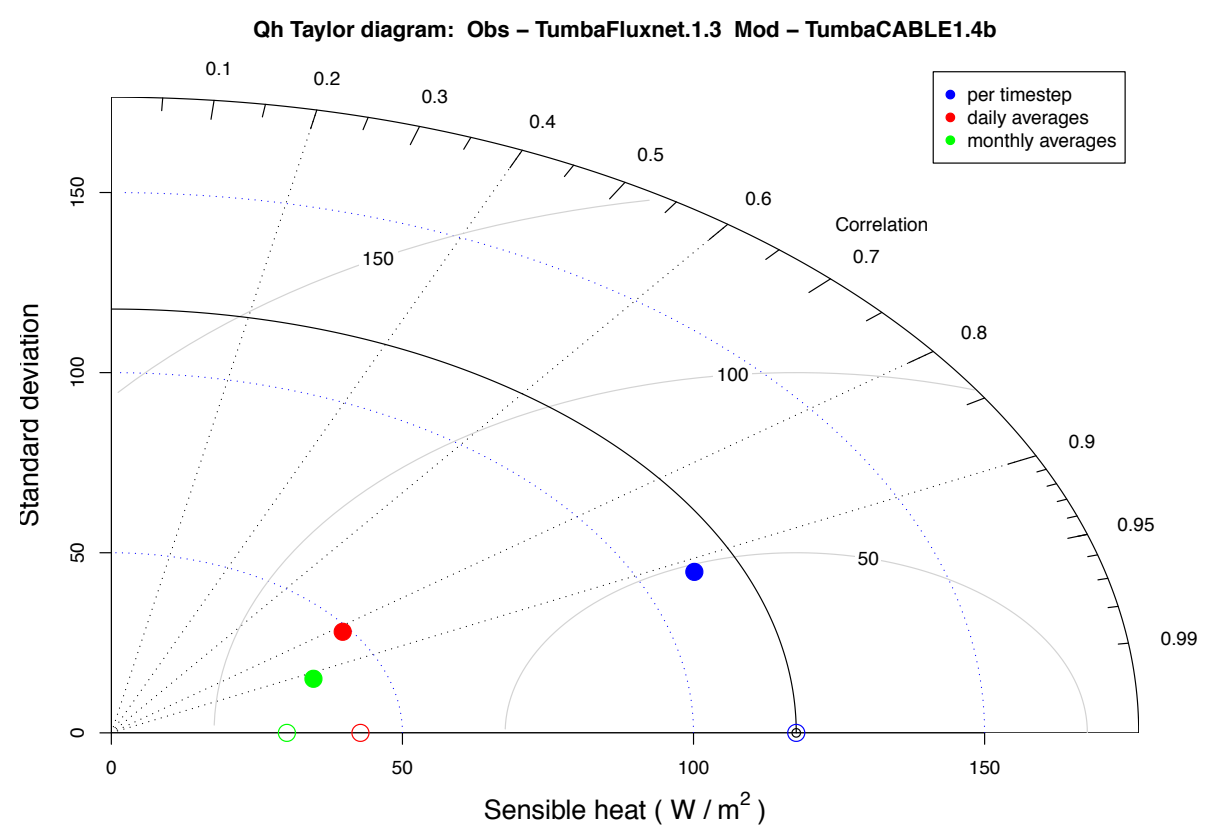

Fig. 4. Taylor diagram of sensible heat flux at a single flux tower site. Per-timestep standard deviation, correlation with observations and root mean square error are shown in blue; daily values in red; and monthly values in green.

PALS R package containing all analysis scripts is available upon request.

When model output files are uploaded, they may be labeled as either "public" or "private". Analyses of "private" outputs are available only to the submitting user, who then effectively uses PALS as a private model development tool. They might continue to upload new model simulations and assess them on PALS without ever sharing results. Alternatively, analyses of "public" model outputs are available to all PALS users. While not yet implemented, a structure to allow a higher-order set of analyses associated with each experiment is being developed. These show the aggregate behaviour of all public model simulations associated with a given experiment, somewhat like an automated, ongoing Project for the Intercomparison of Land-surface Parameterization Schemes (PILPS; Henderson-Sellers et al., 1996) experiment.

Where possible, a single scalar metric is associated with each analysis type. This is intended to aid decision making when comparing two or more model versions across a wide range of metrics. While not yet implemented, a report generation facility is in development to give a multiple-page document summarizing metrics from several models or model versions, specifically for this purpose.

Additionally, PALS allows users to nominate up to three benchmark time series to help evaluate the performance of their LSM output. These can be toggled on/off most existing analysis graphs, with scalar metrics shown for benchmarks in addition to model results. By default, these three benchmarks are three empirical models, as described in Sect. 3, applied to the user's current experiment. As well as empirical models, public LSM outputs from any user associated with the same experiment can also be nominated as benchmarks, although this extension of a priori benchmarking is yet to be implemented.

All of the above features can be accessed in PALS using two different modes. The first is simply within the main PALS database, where a "public" LSM output's analyses are available to all users. The second mode is within a PALS workspace. A workspace can be created by any user, who then becomes the workspace owner. The workspace owner can then invite a subset of PALS users to participate in a workspace, and all data sets, models and public LSM outputs are viewable only to the workspace users. Private LSM outputs remain entirely private in both modes.

Phase 1 of PALS' implementation centres on flux tower data, for the reasons described in Sect. 3 above. PALS currently hosts data from more than 50 flux towers in around 20 countries, with all data taken from the Fluxnet La Thuile free-usage release (http://www.fluxdata.org) and some additional quality control and gap-filling performed. Details about additional processing for each site are available at each site's webpage within PALS. Only consecutive whole years of data were considered, and years with large sections of missing meteorological or flux data were not used. Both meteorological driving data and flux evaluation data are available on the PALS site as ALMA formatted netcdf files.

Currently, the complete analysis set on single flux tower experiments includes the generation of around 50 graphs across 6 LSM output variables and takes around one minute of server processing time to complete. These graphs include: probability density function overlap with observations; 
smoothed time series; model vs. observed scatter plots; Taylor diagrams; seasonally discrete average diurnal cycles; average annual cycle; smoothed evaporative fraction; and conservation of energy checks. Most include a scalar metric as described above. Where data and model output are available, these analyses operate on: net radiation; net shortwave radiation; latent heat flux; sensible heat flux; ground heat flux; and net ecosystem exchange of $\mathrm{CO}_{2}$. Benchmarks in Phase 1 are restricted to comparison with prescribed empirical model time series.

In addition to these LSM-focused features, Phase 1 of PALS maintains the ability for flux tower investigators to directly maintain their data on the PALS site. When a new flux tower data set version is uploaded, PALS runs a suite of automated analysis scripts that explore the properties of uploaded data, including energy conservation and the timing of gap-filling, where meta-data has been included. Data sets are uploaded in a standardized spreadsheet format.

Phase 2 of PALS is likely to include coarse gridded global analysis of variables such as albedo, snow cover, runoff from a selection of catchments globally, as well as a comparison of continental-scale water and carbon budgets. Experimental protocols for these are being developed through the International Land-Atmosphere Model Benchmarking project (www.ilamb.org).

While PALS is still in development as a community-based project, feedback of any nature is welcomed. Contributions in the form of additional analyses, features, or programming support (in R, Java or Flash) are actively encouraged. Both the analysis and website code are available on request through palshelp at gmail dot com.

\section{Conclusions}

The importance of both international standardization of LSM evaluation and the definition of a priori performance benchmarking was illustrated. In particular, it was shown that apparently excellent LSM performance may in fact be poor, and that without quantitative understanding of what should be expected of a LSM in a given experiment, qualitative comparisons may give very little insight. Finally, a community-based automated online evaluation tool, the Protocol for Analysis of Land Surface models (PALS), that attempts to address both of these issues was introduced.

Acknowledgements. PALS is supported by the Australian Terrestrial Ecosystem Research Network (TERN) and the University of New South Wales. It has been developed in cooperation with the Global Energy and Water Cycle Experiment's Global LandAtmosphere System Study (GLASS) panel. This work used eddy covariance data acquired by the FLUXNET community and in particular by the following networks: AmeriFlux (US Department of Energy, Biological and Environmental Research, Terrestrial Carbon Program (DEFG0204ER63917 and DEFG0204ER63911)),
AfriFlux, AsiaFlux, CarboAfrica, CarboEuropeIP, CarboItaly, CarboMont, ChinaFlux, FluxnetCanada (supported by CFCAS, NSERC, BIOCAP, Environment Canada, and NRCan), GreenGrass, KoFlux, LBA, NECC, OzFlux, TCOSSiberia, USCCC. We acknowledge the financial support to the eddy covariance data harmonization provided by CarboEuropeIP, FAOGTOSTCO, iLEAPS, Max Planck Institute for Biogeochemistry, National Science Foundation, University of Tuscia, Université Laval and Environment Canada and US Department of Energy and the database development and technical support from Berkeley Water Center, Lawrence Berkeley National Laboratory, Microsoft Research eScience, Oak Ridge National Laboratory, University of California Berkeley, University of Virginia.

Edited by: D. Lawrence

\section{References}

Abramowitz, G., Leuning, R., Clark, M., and Pitman, A. J.: Evaluating the performance of land surface models, J. Climate, 21, 5468-5481, 2008.

Blyth, E. M., Best, M., Cox, P., Essery, R., Boucher, O., Harding, R., Prentice, I. C., Vidale, P.-L., and Woodward, I.: JULES: a new community land surface model, IGBP newsletter, 6, 9-11, 2006.

Blyth, E. M., Clark, D. B., Ellis, R., Huntingford, C., Los, S., Pryor, M., Best, M., and Sitch, S.: A comprehensive set of benchmark tests for a land surface model of simultaneous fluxes of water and carbon at both the global and seasonal scale, Geosci. Model Dev., 4, 255-269, doi:10.5194/gmd-4-255-2011, 2011.

Collins, W. D., Bitz, C. M., Blackmon, M. L., Bonan, G. B., Bretherton, C. S., Carton, J. A., Chang, P., Doney, S. C., Hack, J. J., Henderson, T. B., Kiehl, J. T., Large, W. G., McKenna, D. S., Santer, B. D., and Smith R. D.: The Community Climate System Model version 3 (CCSM3), J. Climate, 19, 2122-2143, 2006.

Henderson-Sellers, A., McGuffie, K., and Pitman A. J.: The Project for Intercomparison of Land-surface Parameterization Schemes (PILPS): 1992 to 1995, Clim. Dynam., 12, 849-859, 1996.

Kidston, J., Brümmer, C., Black, T. A., Morgenstern, K., Nesic, Z., McCaughey, J. H., and Barr, A. G.: Energy Balance Closure Using Eddy Covariance Above Two Different Land Surfaces and Implications for $\mathrm{CO}_{2}$ Flux Measurements, Bound.-Lay. Meteorol., 136, 193-218, doi:10.1007/s10546-010-9507-y, 2010.

Ginzburg, L. R. and Jensen, C. X. J.: Rules of thumb for judging ecological theories, Trends Ecol. Evol., 19, 121-126, 2004.

Gordon, H. B., Rotstayn, L. D., McGregor, J., Dix, M. R., Kowalczyk, E. A., Farrell, S. P. O., Waterman, L. J., Hirst, A. C., Wilson, S. G., Collier, M. A., Watterson, I. G., and Elliott., T. I.: The CSIRO Mk3 climate system model, Technical Report 60, CSIRO Atmospheric Research, Aspendale, Melbourne, 2002.

Krinner, G., Viovy, N., de Noblet-Ducoudre, N., Ogee, J., Polcher, J., Friedlingtein, P., Ciais, P., Sitch, S., and Prentice I. C.: A dynamic global vegetation model for studies of the coupled atmosphere-biosphere system, Global Biogeochem. Cy., 19, GB1015, doi:10.1029/2003GB002199, 2005.

Leuning, R., Cleugh, H. A., Zegelin, S. J., and Hughes, D.: Carbon and water fluxes over a temperate Eucalyptus forest and a tropical wet/dry savanna in Australia: Measurements and comparison 
with MODIS remote sensing estimates, Agr. Forest Meteorol., 129, 151-173, 2005.

Levis, S., Bonan, G., Vertenstein, M., and Oleson, K.: The community land model's dynamic global vegetation model (CLMDGVM): Technical description and user's guide, NCAR Tech. Rep. TN-459+IA, 50 pp., 2004.

Manabe, S.: Climate and the ocean circulation: 1, the atmospheric circulation and the hydrology of the Earth's surface, Mon. Weather Rev., 97, 739-805, 1969.

Medlyn, B. E., Robinson, A. P., Clement, R., and McMurtrie, R. E.: On the validation of models of forest $\mathrm{CO}_{2}$ exchange using eddy covariance data: Some perils and pitfalls, Tree Physiol., 25, 839-857, 2005

Oleson, K. W., Dai, Y., Bonan, G., Bosilovich, M., Dickinson, R., Dirmeyer, P., Hoffman, F., Houser, P., Levis, S., Niu, G.-Y., Thornton, P., Vertenstein, M., Yang, Z.-L., and Zeng, X.: Technical description of the community land model (CLM), NCAR Tech. Rep., TN-461+STR, 174 pp., 2004.
Oreskes, N., Shrader-Frechette, K., and Belitz, K.: Verification, validation, and confirmation of numerical models in the earth sciences, Science, 263, 641-646, doi:10.1126/science.263.5147.641, 1994.

Wang, Y. P., Kowalczyk, E., Leuning, R., Abramowitz, G., Raupach, M. R., Pak, B., van Gorsel, E., and Luhar, A.: Diagnosing errors in a land surface model (CABLE) in the time and frequency domains, J. Geophys. Res., 116, G01034, doi:10.1029/2010JG001385, 2011.

Wilson, K., Goldstein, A., Falge, E., Aubinet, M., Baldocchi, D., Berbigier, P., Bernhofer, C., Ceulemans, R., Dolman, H., Field, C., Grelle, A., Ibrom, A., Law, B. E., Kowalski, A., Meyers, T., Moncrieff, J., Monson, R., Oechel, W., Tenhunen, J., Valentini, R., and Verma, S.: Energy balance closure at FLUXNET sites, Agr. Forest Meteorol., 113, 223-243, 2002. 